\title{
Intrapericardial bevacizumab safely and effectively treats malignant pericardial effusion in advanced cancer patients
}

\author{
Dawei Chen ${ }^{1,2, *}$, Yan Zhang ${ }^{2,3, *}$, Fang Shi ${ }^{2,3}$, Hui Zhu ${ }^{2,3}$, Minghuan Li2,3, Kaijun Chen ${ }^{4}$, \\ Li Kong ${ }^{2,3}$ and Jinming $\mathbf{Y u}^{2,3}$ \\ ${ }^{1}$ Weifang Medical University, Weifang, China \\ ${ }^{2}$ Department of Radiation Oncology, Shandong Cancer Hospital affiliated to Shandong University, Jinan, China \\ ${ }^{3}$ Shandong Academy of Medical Sciences, Jinan, China \\ ${ }^{4}$ Department of Clinical Laboratory, Mengyin People's Hospital, Linyi, China \\ * These authors have contributed equally to this work \\ Correspondence to: Li Kong, email: Kongli7@sina.com
}

Jinming Yu, email: sdyujinming@163.com

Keywords: bevacizumab, intrapericardial, malignant pericardial effusion, advanced cancer

Received: January 14, $2016 \quad$ Accepted: May 09, 2016

Published: May 17, 2016

\section{ABSTRACT}

We evaluated the safety and efficacy of intrapericardial bevacizumab (BEV) for treating symptomatic malignant pericardiac effusion (MPCE) in seven advanced cancer patients. All patients had previously undergone multiple lines of systemic therapy. Each patient received paracentesis and intrapericardial infusions of 100 or $200 \mathbf{~ m g}$ of BEV every two weeks. Systemic treatments for primary tumors continued for all patients during BEV treatment. Of the seven patients, three achieved a complete response, two achieved a partial response, and two showed no response with regard to MPCE after BEV infusion. The median overall survival time was 168 days (range, 22224 days). In six of the seven patients, effusion did not recur before death. Toxicity associated with BEV treatment was mild and manageable in all patients. This study provides preliminary evidence that intrapericardial BEV may be an effective and safe treatment for MPCE in patients with advanced cancers.

\section{INTRODUCTION}

Malignant pericardial effusion (MPCE), a common complication that causes refractory cardiac dysfunction and chronic pericardial tamponade, affects up to $15 \%$ of advanced cancer patients and shortens their overall survival [1, 2]. MPCE develops rapidly and is lifethreatening, requiring prompt and effective salvage therapy [3]. Current clinical treatments for MPCE include indwelling pericardial catheters, fenestrated drainage, and intrapericardial infusion of therapeutic agents [4]. However, the efficacy of these treatments is poor, and effusion eventually reoccurs $[5,6]$. Therefore, in order to improve patients' quality of life, more effective therapies for MPCE are necessary.

Recent studies showed that intrapericardial infusion of bevacizumab (BEV) was an effective treatment for malignant ascites and pleural effusion $[7,8,9,10]$. Here, we examined the effects of BEV in advanced cancer patients with MPCE.

\section{MATERIALS AND METHODS}

Patients

Seven advanced cancer patients with symptomatic MPCE were included in our retrospective study. All patients received intrapericardial infusion of $\mathrm{BEV}$ at Shandong Cancer Hospital between May 2011 and November 2013. Advanced cancer was diagnosed by pathology. MPCE was detected using ultrasonography and computed tomography and confirmed by cytology. The median age of the patients was 45 years (range, 23-75 years). Four patients had stage IV lung cancer, and the remaining three patients had esophageal cancer, malignant mesothelioma of the pleura, and a mediastinal yolk sac tumor. Typical cardiac tamponade symptoms were observed in all patients, including dyspnea in seven patients, stethalgia in six patients, and tachycardia in five 
patients. All patients had previously undergone multiple lines of systemic therapy. Frequent paracentesis was required in order to relieve cardiac tamponade symptoms. Table 1 summarizes the clinical characteristics of the patients. This study was approved by the Shandong Cancer Hospital Institutional Review Board.

\section{Intrapericardial infusion of BEV}

All patients required pericardial catheterization with the aid of ultrasound. Each patient received drainage more than one time to remove pericardial effusions as much as possible. When no obvious effusion remained, patients received an intrapericardial infusion of 100 or $200 \mathrm{mg}$ of $\mathrm{BEV}$ diluted in $30 \mathrm{~mL}$ of physiological saline. To ensure the uniform distribution of bevacizumab in the pericardial cavity, patients were advised to turn over smoothly every $15 \mathrm{~min}$. This treatment was repeated every two weeks until a response was observed.

\section{Concurrent systemic therapy}

In addition to $\mathrm{BEV}$ pericardial infusion, the patients continued to receive systemic therapies to treat their primary tumors. Two patients with confirmed EGFR gene mutations received concurrent erlotinib (150 mg every day). Four patients received systemic chemotherapy that included $75 \mathrm{mg} / \mathrm{m}^{2}$ cisplatin on days 1,2 , and 3 , and either $500 \mathrm{mg} / \mathrm{m}^{2}$ pemetrexed on day 1 or $1000 \mathrm{mg} / \mathrm{m}^{2}$ gemcitabine on days 1 and 8 (Table 2). All chemotherapy cycles were repeated every 3 weeks for 2-4 cycles. Patients who did not show progression after treatment with the above regimens received pemetrexed or gemcitabine maintenance therapy every 3 weeks until disease progression. One patient with an Eastern Cooperative Oncology Group (ECOG) score of 4 could not tolerate systemic chemotherapy and received supportive treatment exclusively.

\section{Evaluation of intrapericardial infusion of BEV}

Ultrasonography and computed tomography were used to evaluate the therapeutic efficacy of intrapericardial infusion of BEV for MPCE. According to previous studies $[8,9,10]$ and the Response Evaluation Criteria in Solid Tumors (RECIST, version 1.1), therapeutic efficacy of BEV for MPCE was classified as follows: 1) complete response, MPCE completely disappeared within 4 weeks; 2) partial response, MPCE was reduced more than $50 \%$ within 4 weeks; or 3) no response, MPCE was reduced by less than $50 \%$ or effusion increased. Effusion-free survival time was used to evaluate the duration of the local pericardial response. To assess adverse events, we used the National Cancer Institute Common Terminology Criteria for Adverse Events grading system (CTCAE, version 4.0).

\section{RESULTS}

\section{Efficacy}

One of the seven patients completed one cycle, five patients received two cycles and one patient completed three cycles of pericardiocentesis with BEV infusion. The drainage catheter was successfully removed from all patients. Noticeable remission of cardiac tamponade symptoms was observed in six patients, and typical symptoms such as dyspnea, stethalgia, and tachycardia did not recur during the follow-up period. Three patients achieved complete response (Figures 1 and 2), two patients achieved partial response, and two patients had no response. In six of the patients, effusion did not reoccur before death, and effusion-free survival times ranged from 22 to 224 days (median, 168 days). Only one patient experienced effusion recurrence (Table 2).

\section{Drug-related adverse events}

All drug-related adverse events experienced by patients were mild and manageable (Table 3 ). The common side effects that accompany systemic chemotherapy, including hematological toxicity, hepatotoxicity, and nephrotoxicity, were not observed. Two patients experienced nausea and vomiting. One patient experienced mild proteinuria related to bevacizumab treatment. None of the patients experienced hypertension. In addition, one patient experienced thrombosis in both legs, accompanied by abnormal clotting, which was resolved by anticoagulation treatment. None of the patients discontinued treatment because of serious adverse events.

\section{DISCUSSION}

MPCE is a common complication in advanced cancer patients and is associated with very poor prognosis, and current treatments for MPCE are not effective $[5,6]$. Additionally, the mechanisms underlying MPCE are poorly understood. According to previous studies, MPCE usually results from lymphatic obstruction due to the spread of cancer into draining lymph vessels [11, 12, 13, 14]. Angiogenesisis may contribute to the production of malignant effusion $[15,16,17]$, and tumors often overexpress vascular endothelial growth factor (VEGF), which is a major pro-angiogenic factor [18]. VEGF can cause tumor vessels to become disorganized, leaky, and tortuous, and can promote the production of malignant effusions in serous cavities such as the pericardial cavity $[19,20,21]$.

Because the development of effusion likely 
Table 1: Clinical characteristics of patients with malignant pericardial effusion

\begin{tabular}{|c|c|c|c|c|c|c|c|c|c|}
\hline $\begin{array}{l}\text { Patient } \\
\text { No. }\end{array}$ & $\begin{array}{l}\text { Primary } \\
\text { tumor }\end{array}$ & Gender & Age & Pathological type & Stage & $\begin{array}{l}\text { ECOG } \\
\text { Score }\end{array}$ & Pre -systemic therapy & $\begin{array}{l}\text { Pre- } \\
\text { intrapericardial } \\
\text { infusion }\end{array}$ & Symptoms \\
\hline 1 & Lung cancer & $\mathrm{M}$ & 38 & Adenocarcinoma & $\mathrm{pT}_{4} \mathrm{~N}_{1} \mathrm{M}_{1}$ & 2 & $\begin{array}{l}\text { Pneumonectomy, } \\
\text { chemotherapy, TKI }\end{array}$ & IL-2, CDDP & Dyspnea, Stethalgia, \\
\hline 2 & Lung cancer & M & 60 & Adenocarcinoma & $\mathrm{cT}_{4} \mathrm{~N}_{2} \mathrm{M}_{1}$ & 2 & Chemoradiotherapy & CDDP & $\begin{array}{l}\text { Dyspnea, Stethalgia, } \\
\text { Tachycardia }\end{array}$ \\
\hline 3 & Lung cancer & $\mathrm{F}$ & 68 & Adenocarcinoma & $\mathrm{cTXNXM}_{1}$ & 2 & Chemoradiotherapy & - & $\begin{array}{l}\text { Dyspnea, Stethalgia, } \\
\text { Tachycardia }\end{array}$ \\
\hline 4 & Lung cancer & $\mathrm{F}$ & 40 & Adenocarcinoma & $\mathrm{cT}_{4} \mathrm{~N}_{2} \mathrm{M}_{1}$ & 3 & $\begin{array}{l}\text { Chemoradiotherapy, } \\
\text { TKI }\end{array}$ & - & $\begin{array}{l}\text { Dyspnea, Stethalgia, } \\
\text { Tachycardia }\end{array}$ \\
\hline 5 & $\begin{array}{l}\text { Esophageal } \\
\text { cancer }\end{array}$ & M & 75 & Squamous & $\mathrm{pT}_{3} \mathrm{~N}_{2} \mathrm{M}_{1}$ & 1 & Chemotherapy & CDDP & Dyspnea \\
\hline 6 & MMP & M & 45 & Epithelial type & $\mathrm{cT}_{4} \mathrm{~N} 3 \mathrm{M}_{1}$ & 4 & Chemotherapy, TKI & - & $\begin{array}{l}\text { Dyspnea, Stethalgia, } \\
\text { Tachycardia }\end{array}$ \\
\hline 7 & $\begin{array}{l}\text { Mediastinal } \\
\text { tumors }\end{array}$ & M & 23 & Yolk sac tumor & $\mathrm{cT}_{4} \mathrm{~N}_{2} \mathrm{M}_{1}$ & 4 & Chemotherapy & CDDP & $\begin{array}{l}\text { Dyspnea, Stethalgia, } \\
\text { Tachycardia }\end{array}$ \\
\hline
\end{tabular}

Abbreviations: MMP, malignant mesothelioma of pleura; M, male; F, female; ECOG, Eastern ; Cooperative Oncology Group; TKI, tyrosine kinase inhibitor; BEV, bevacizumab; CDDP, cisplatin; IL-2, interleukin-2

Table 2: Protocols for intrapericardial infusion of BEV and response to therapy

\begin{tabular}{|l|l|l|l|l|l|l|}
\hline Patient No. & BEV infusion (mg) $\times$ times & $\begin{array}{l}\text { Interval times } \\
\text { (days) }\end{array}$ & $\begin{array}{l}\text { Concurrent systemic } \\
\text { therapy }\end{array}$ & Response & EFS(days) & Survival(days) \\
\hline 1 & $100 \times 2$ & 14 & Erlotinib & CR & 196 & 196 \\
\hline 2 & $200 \times 2$ & 14 & PEX + CDDP & CR & 168 & 168 \\
\hline 3 & $200 \times 2$ & 14 & PEX + CDDP & PR & 102 & 102 \\
\hline 4 & $100 \times 3$ & 14 & Erlotinib & CR & 224 & 224 \\
\hline 5 & $100 \times 2$ & 14 & GEM+ CDDP & PR & 182 & 182 \\
\hline 6 & $200 \times 1$ & 14 & GEM+ CDDP & NR & 22 & 22 \\
\hline 7 & $200 \times 2$ & 14 & - & NR & 40 & 46 \\
\hline
\end{tabular}

Abbreviations: BEV, bevacizumab; CR, complete response; PR, partial response; NR, no response;

GEM, gemcitabine; PEX, pemetrexed; CDDP, cisplatin; EFS, effusion-free survival time.

Table 3: Drug-related adverse events for bevacizumab intrapericardial infusion (CTCAE version 4.0)

\begin{tabular}{|l|l|l|l|l|l|l|l|l|}
\hline $\begin{array}{l}\text { Patient } \\
\text { No. }\end{array}$ & Proteinuria & Thrombus & Hypertension & $\begin{array}{l}\text { Nausea } \\
\text { vomiting }\end{array}$ & Hemorrhage & Hematological toxicity & Hepatotoxicity & Nephrotoxicity \\
\hline 1 & - & - & - & G1 & & - & - & - \\
\hline 2 & G1 & - & - & - & & - & - & - \\
\hline 3 & - & G2 & - & - & & - & - & - \\
\hline 4 & - & - & - & - & & - & - & - \\
\hline 5 & - & - & - & - & & - & - & - \\
\hline 6 & - & & - & G2 & & - & - & - \\
\hline 7 & - & - & - & - & & - & - & - \\
\hline
\end{tabular}

Abbreviations: $\mathrm{G} 1$ and $\mathrm{G} 2$, grade of adverse events in patients according to CTCAE, the National

Cancer Institute Common Terminology Criteria for Adverse Event.

results from abnormal vascularity and the permeability of tumors $[22,23,24,25,26]$, anti-VEGF agents might be effective in treating MPCE. BEV, which blocks the binding of human VEGF to its receptors, is a recombinant humanized monoclonal anti-VEGF antibody [27]. In preclinical studies, BEV inhibited the growth of 13 types of malignant cells [28]. Additionally, it decreased the density, diameter, and permeability of vessels, consequently reducing interstitial fluid pressure [22]. In animal models $[22,23]$ and clinical studies $[7,8,9], \mathrm{BEV}$ inhibited the development of malignant effusions in serous cavities more safely and effectively than commonly used cytotoxic agents, such as cisplatin and 5-fluorouracil. There are very few reports investigating intrapericardial $\mathrm{BEV}$ as a treatment for MPCE. In a previous exploratory study, we infused BEV into the pericardium of a lung cancer patient with MPCE and achieved complete response, with the patient remaining free of fluid for five months [29]. Given this encouraging result, we repeated this treatment here in seven patients with refractory MPCE to confirm its efficacy. The median survival time of these patients was 168 days (range, 22-224 days). 
All patients achieved obvious and sustained symptom remission, and six of the seven patients did not suffer from effusion recurrence before death. Similar studies using intrapericardial cisplatin reported median survival times of $84 \pm 39$ days [30] and $120 \pm 71$ days (range, 68-268 days) [31]. Our results in this group of patients suggest that intrapericardial BEV may be more effective than other therapies in treating MPCE.

Among the patients in our study, two had point mutations in exon 21 of the EGFR gene and developed MPCE during erlotinib treatment. Both of these patients received intrapericardial infusions of BEV while continuing with the initial erlotinib regimen. These patients, who did not receive any additional systemic therapies, had a longer remission period than the other patients. Similar to previous data [32], these results suggest that both EGFR gene mutations and erlotinib efficacy may be associated with better prognoses. Moreover, BEV perfusion might be especially beneficial for MPEC patients who experience disease progression during erlotinib treatment. For lung cancer patients with MPEC and EGFR gene mutations, intrapericardial BEV might therefore be particularly effective.

We found that intrapericardial BEV was a safe and effective treatment for MPCE, and toxicities associated with this treatment were mild and endurable. Maisch et al. [30] and Oida et al. [31] reported nausea in most patients who received cisplatin infusions. In contrast, no hematological toxicity, hepatotoxicity, or nephrotoxicity was observed in any of the patients in our study after $\mathrm{BEV}$ treatment. Bilateral thrombosis was observed in one patient who had a history of atherosclerosis and myocardial infarctions and an elevated blood level of D-dimer before treatment, all of which are associated with

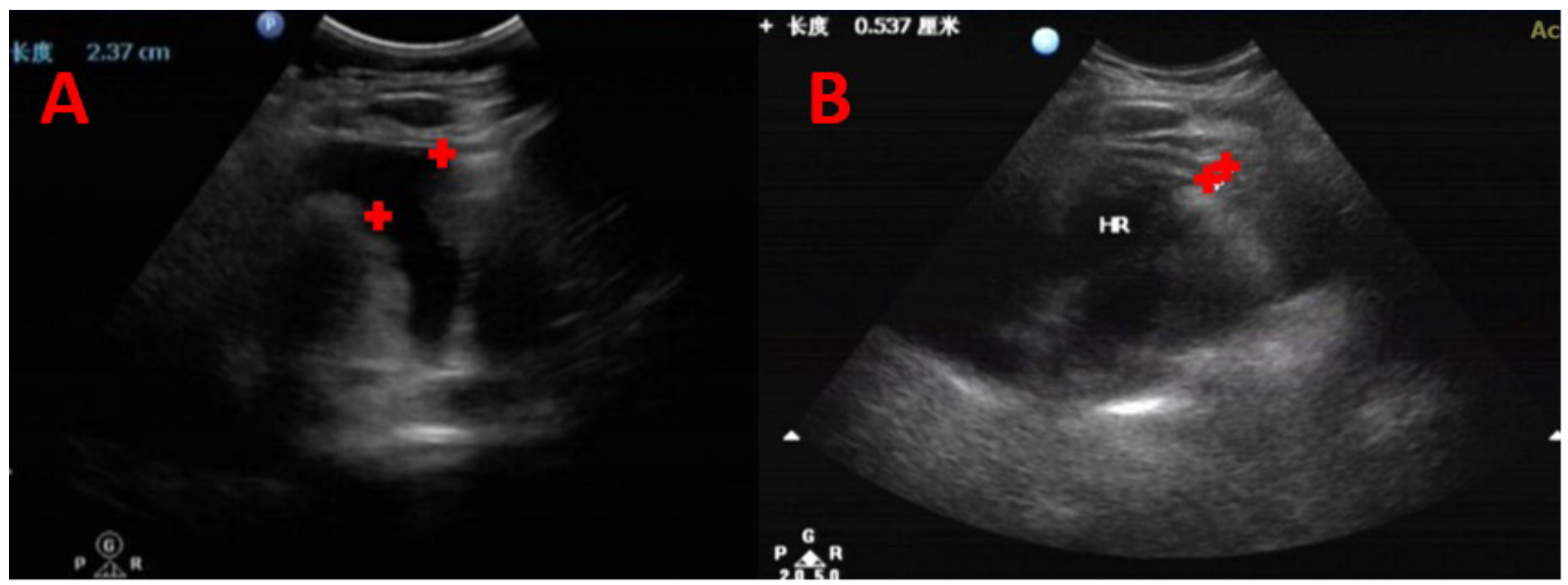

Figure 1: B-ultrasonography images of a 60-year-old man with advanced lung cancer who had recurrent malignant pericardial effusion (MPCE) and who had received multi-line therapy to control MPCE with unsatisfactory outcomes. MPCE almost completely disappeared after bevacizumab perfusion until his death. (A) Five days before bevacizumab intrapericardial infusion. (B) Four months after bevacizumab intrapericardial infusion

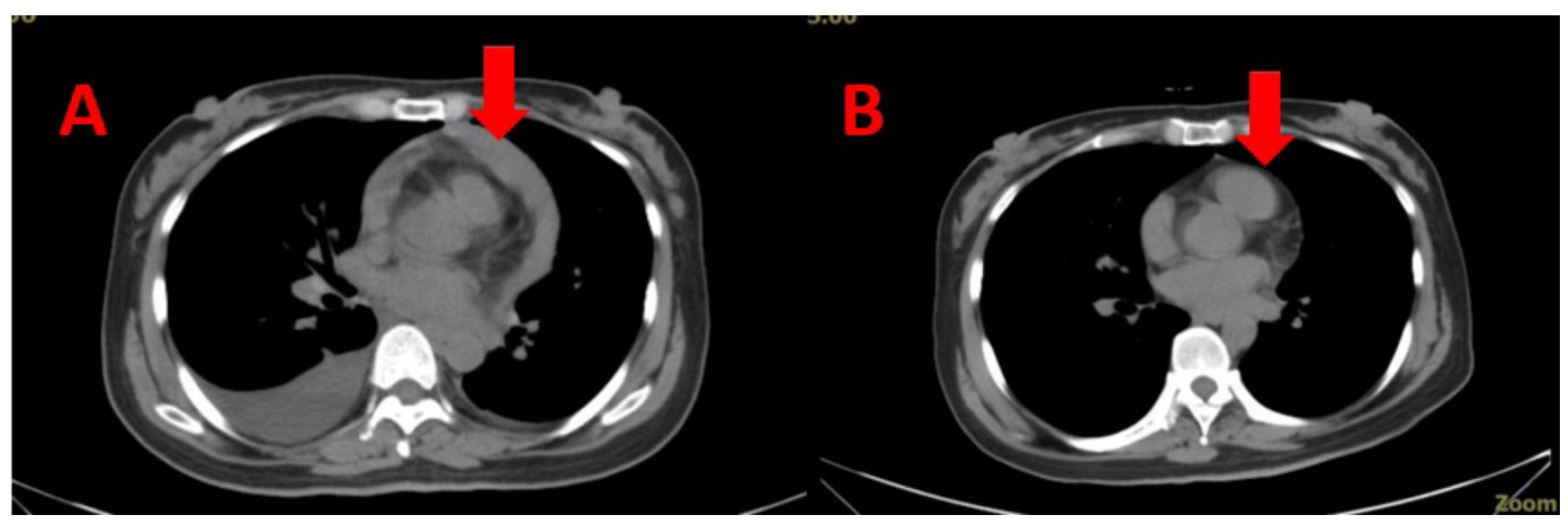

Figure 2: Computed tomography images of a 40-year-old woman with advanced lung cancer who had recurrent malignant pericardial effusion (MPCE) and had received chemoradiotherapy and tyrosine kinase inhibitor therapy without a satisfactory outcome. However, bevacizumab infusion was effective. (A) Two days before bevacizumab intrapericardial infusion. (B) Six months after bevacizumab intrapericardial infusion. 
high risk for bilateral thrombosis. Intravenous infusion of BEV has been shown to cause thrombosis [7, 21], but it remains unclear whether intrapericardial infusion of BEV has the same effect. Although the occurrence of thrombosis was not necessarily due to BEV infusion, patients with abnormal clotting conditions who receive this treatment should be closely monitored.

In previous studies, the doses of BEV used to treat ascites and pleural effusion were $15 \mathrm{mg} / \mathrm{kg}$ and $7.5 \mathrm{mg} /$ $\mathrm{kg}[8,34]$. To date, there is little information regarding the most appropriate dosage of $\mathrm{BEV}$ for intrapericardial perfusion. In our study, most of the patients received a $200 \mathrm{mg}$ dose. This dosage was on the high end of the previously investigated dose range $(7.5 \mathrm{mg} / \mathrm{kg}-15 \mathrm{mg} /$ $\mathrm{kg}$ ). Additionally, three patients received $100 \mathrm{mg}$ of BEV due to poor ECOG scores (two patients) or advanced age (75 years, one patient). However, this lower dose was still effective in treating MPCE. Because of the small number of patients in our study, we were unable to determine the most appropriate dosage of BEV.

The results of this study provide early evidence that intrapericardial BEV may be an effective and safe treatment for MPCE arising from various malignancies. Further studies are required to investigate the side effects of this treatment and the appropriate BEV dosage. These studies should include larger patient cohorts to allow for appropriate statistical analyses.

\section{ACKNOWLEDGMENTS}

This work was supported by the National Natural Science Foundation of China (81402518), the Natural Science Foundation of Jiangsu (BK20151174), and the Wu Jieping Medical Foundation (320.6750.14021).

\section{CONFLICTS OF INTEREST}

The authors report no conflicts of interest for this work.

\section{REFERENCES}

1. Hanafy AF, El-Egaky AM, Mortada SA, Molokhia AM. Development of implants for sustained release of 5-fluorouracil using low molecular weight biodegradable polymers. Drug Discoveries and Therapeutics. 2009; 3: 287-295.

2. McAllister HA, Jr., Hall RJ, Cooley DA. Tumors of the heart and pericardium. Current Problems in Cardiology. 1999; 24: 57-116.

3. Skhvatsabaja LV. Secondary malignant lesions of the heart and pericardium in neoplastic disease. Oncology. 1986; 43: 103-106.

4. Martinoni A, Cipolla CM, Civelli M, Cardinale D, Lamantia G, Colleoni M, DeBraud F, Susini G, Martinelli
G, Goldhirsh A, Fiorentini C. Intrapericardial treatment of neoplastic pericardial effusions. Herz. 2000; 25: 787-793.

5. Kats S, Nieuwenhuijzen GA, van Straten BH, Schonberger JP. Cardiac tamponade: an unusual, lifethreatening complication after transhiatal resection of the esophagus. Interactive Cardiovascular and Thoracic surgery. 2007; 6: 238-239.

6. Mizuguchi Y, Takeda S, Miyashita M, Ikezaki H, Nakajima Y, Akada S, Makino H, Futami R, Arai M, Sasajima K, Tajiri T, Tanaka K. A case of cardiac tamponade following esophageal resection. Journal of Anesthesia. 2005; 19: 249251.

7. Hamilton CA, Maxwell GL, Chernofsky MR, Bernstein SA, Farley JH, Rose GS. Intraperitoneal bevacizumab for the palliation of malignant ascites in refractory ovarian cancer. Gynecologic Oncology. 2008; 111: 530-532.

8. Du N, Li X, Li F, Zhao H, Fan Z, Ma J, Fu Y, Kang H. Intrapleural combination therapy with bevacizumab and cisplatin for non-small cell lung cancer-mediated malignant pleural effusion. Oncology Reports. 2013; 29: 2332-2340.

9. Numnum TM, Rocconi RP, Whitworth J, Barnes MN. The use of bevacizumab to palliate symptomatic ascites in patients with refractory ovarian carcinoma. Gynecologic Oncology. 2006; 102: 425-428.

10. Jiang L, Li P, Gong Z, Hu B, Ma J, Wang J, Chu H, Zhang L, Sun P, Chen J. Effective treatment for malignant pleural effusion and ascites with combined therapy of bevacizumab and cisplatin. Anticancer Research. 2016; 36: 1313-1318.

11. Chernow B, Sahn SA. Carcinomatous involvement of the pleura: an analysis of 96 patients. The American Journal of Medicine. 1977; 63:695-702.

12. Feldman GB, Knapp RC. Lymphatic drainage of the peritoneal cavity and its significance in ovarian cancer. American Journal of Obstetrics and Gynecology. 1974; 119: 991-994.

13. Meyer PC. Metastatic carcinoma of the pleura. Thorax. 1966; 21: 437-443.

14. Feldman GB, Knapp RC, Order SE, Hellman S. The role of lymphatic obstruction in the formation of ascites in a murine ovarian carcinoma. Cancer Research. 1972; 32: 1663-1666.

15. Heuser LS, Taylor SH, Folkman J. Prevention of carcinomatosis and bloody malignant ascites in the rat by an inhibitor of angiogenesis. The Journal of Surgical Research. 1984; 36: 244-250.

16. Folkman J. Tumor angiogenesis. Advances in Cancer Research. 1974; 19: 331-358.

17. Klagsbrun M, Knighton D, Folkman J. Tumor angiogenesis activity in cells grown in tissue culture. Cancer Research. 1976; 36: 110-114.

18. Zhou WB, Bai M, Jin Y. Diagnostic value of vascular endothelial growth factor and endostatin in malignant pleural effusions. International Journal of Tuberculosis and Lung Disease. 2009; 13: 381-386. 
19. Gasparini G, Toi M, Gion M, Verderio P, Dittadi R, Hanatani M, Matsubara I, Vinante O, Bonoldi E, Boracchi P, Gatti C, Suzuki H, Tominaga T. Prognostic significance of vascular endothelial growth factor protein in nodenegative breast carcinoma. Journal of the National Cancer Institute. 1997; 89: 139-147.

20. Fontanini G, Vignati S, Boldrini L, Chine S, Silvestri V, Lucchi M, Mussi A, Angeletti CA and Bevilacqua G. Vascular endothelial growth factor is associated with neovascularization and influences progression of non-small cell lung carcinoma. Clinical Cancer Research. 1997; 3: 861-865.

21. Tang N, Guo J, Zhang Q, Wang Y, Wang Z. Greater efficacy of chemotherapy plus bevacizumab compared to chemoand targeted therapy alone on non-small cell lung cancer patients with brain metastasis. Oncotarget. 2016; 7: 36353644. doi: 10.18632/oncotarget.6184.

22. Ribeiro SC, Vargas FS, Antonangelo L, Marchi E, Genofre EH, Acencio MM, Teixeira LR. Monoclonal anti-vascular endothelial growth factor antibody reduces fluid volume in an experimental model of inflammatory pleural effusion. Respirology. 2009; 14: 1188-1193.

23. Luo JC, Yamaguchi S, Shinkai A, Shitara K, Shibuya M. Significant expression of vascular endothelial growth factor/vascular permeability factor in mouse ascites tumors. Cancer Research. 1998; 58: 2652-2660.

24. Byrne AT, Ross L, Holash J, Nakanishi M, Hu L, Hofmann JI, Yancopoulos GD, Jaffe RB. Vascular endothelial growth factor-trap decreases tumor burden, inhibits ascites, and causes dramatic vascular remodeling in an ovarian cancer model. Clinical Cancer Research. 2003; 9: 5721-5728.

25. Xu L, Yoneda J, Herrera C, Wood J, Killion JJ, Fidler IJ. Inhibition of malignant ascites and growth of human ovarian carcinoma by oral administration of a potent inhibitor of the vascular endothelial growth factor receptor tyrosine kinases. International Journal of Oncology. 2000; 16: 445-454.

26. Stoelcker B, Echtenacher B, Weich HA, Sztajer H, Hicklin DJ, Mannel DN. VEGF/Flk-1 interaction, a requirement for malignant ascites recurrence. Journal of Interferon and Cytokine Research. 2000; 20: 511-517.
27. Presta LG, Chen H, O'Connor SJ, Chisholm V, Meng YG, Krummen L, Winkler M, Ferrara N. Humanization of an anti-vascular endothelial growth factor monoclonal antibody for the therapy of solid tumors and other disorders. Cancer Research. 1997; 57: 4593-4599.

28. Takahashi Y, Tucker SL, Kitadai Y, Koura AN, Bucana CD, Cleary KR, Ellis LM. Vessel counts and expression of vascular endothelial growth factor as prognostic factors in node-negative colon cancer. Archives of Surgery. 1997; 132: 541-546.

29. Chen D, Zhang Y, Shi F, Li M, Zhu H, Kong L, Yu J. Sustained response of malignant pericardial effusion to intrapericardial bevacizumab in an advanced lung cancer patient: a case report and literature review. OncoTargets and Therapy. 2015; 8: 2767-2770.

30. Maisch B, Ristic AD, Pankuweit S, Neubauer A, Moll R. Neoplastic pericardial effusion. Efficacy and safety of intrapericardial treatment with cisplatin. European Heart Journal. 2002; 23: 1625-1631.

31. Oida T, Mimatsu K, Kano H, Kawasaki A, Kuboi Y, Fukino N, Amano S. Pericardiocentesis with cisplatin for malignant pericardial effusion and tamponade. World Journal of Gastroenterology. 2010; 16: 740-744.

32. Zhou C, Wu YL, Chen G, Liu X, Zhu Y, Lu S, Feng J, He J, Han B, Wang J, Jiang G, Hu C, et al. BEYOND: a randomized, double-blind, placebo-controlled, multicenter, phase III study of first-line carboplatin/paclitaxel plus bevacizumab or placebo in Chinese patients with advanced or recurrent nonsquamous non-small-cell lung cancer. Journal of Clinical Oncology. 2015; 33: 2197-2204.

33. Moja L, Lucenteforte E, Kwag KH, Bertele V, Campomori A, Chakravarthy U, D'Amico R, Dickersin K, Kodjikian L, Lindsley K, Loke Y, Maguire M, Martin DF, et al. Systemic safety of bevacizumab versus ranibizumab for neovascular age-related macular degeneration. Cochrane Database of Systematic Reviews. 2014; 9: CD011230.

34. Chen D, Li X, Zhao H, Fu Y, Yao F, Hu J, Du N. The efficacy of pemetrexed and bevacizumab intrapleural injection for malignant pleural mesothelioma-mediated malignant pleural effusion. Indian Journal of Cancer. 2014; 51: e82-85. 